\title{
EXPERIENCIA DE EDUCACIÓN EN PRODUCCIÓN AGROECOLÓGICA FAMILIAR y DESARROLLO AGROPECUARIO - COMUNIDAD LLAÑUCANCHA - ABANCAY
}

\section{EXPERIENCE OF AGROECOLOGICAL FAMILY EDUCATION AND AGRICULTURAL DEVELOPMENT COMMUNITY LLAÑUCANCHA ABANCAY.}

\author{
Riveros; Antonio \\ tony_rs7@hotmail.com \\ El Autor declara no tener Conflicto de Interés por la publicación del presente Artículo \\ Científico.
}

\section{RESUMEN}

El estudio de investigación, identifico y analizo aspectos educacionales-formativos y de intercambio, dentro de procesos técnico-productivos, sociales, culturales que desarrollan algunos agricultores(as), desde una perspectiva de revaloración de "saberes locales", bajo los enfoques de la producción agroecológica familiar, y la relación e influencia de éstos en su entorno comunal y territorial. Parte del proceso encontró soporte en la aplicación práctica de sus experiencias locales y conocimientos ancestrales, como base para la creación de conocimiento nuevo a nivel familiar, y su transferencia e intercambio desde las familias agroecológicas hacia los propios productores(as) locales, utilizando metodologías de educación andragógica. El estudio tuvo como objetivo analizar el nivel de relación e influencia entre la experiencia de educación en producción agroecológica familiar con los procesos de desarrollo agropecuario de familias rurales, -a nivel de verificación de procesos de intercambio y transferencia de experiencias-. A nivel especifico se tuvo como objetivos establecer estas relaciones a nivel de los procesos de producción agrícola y pecuario.

Para el estudio se aplicó el diseño metodológico: “Correlacional No Experimental”. Para el análisis de las variables, se utilizó Cuestionarios, como instrumentos de recojo de información (26 items de tipo abierto-Valoración Escala Likert). Las unidades de análisis lo conformaron los "productores(as) agroecológicos"; y a su vez, las unidades de muestreo fueron las "unidades agrícolas". Los resultados de la investigación 
demostraron que existe tendencias sobre la predisposición de las familias de productores(as), para la aplicación y/o adopción sistemática de tecnología validada para la producción agroecológica, a través de la creación y conservación de saberes locales, conducentes a procesos de innovación para el desarrollo sostenible de su agricultura y producción pecuaria. Se puede inferir que, entre las variables de estudio existe una relación pertinente ya que en ambos casos la muestra se encuentra en un nivel basado en los procesos productivos actuales que desarrolla la población comunal en estudio.

Palabras clave: Unidad agrícola familiar, agronomía, pecuario.

\begin{abstract}
This research study identifies and analyzes educational-training and exchange aspects, within technical-productive, social, cultural processes that some farmers develop, from a perspective of revaluation of "local knowledge", under the approaches of family agroecological production, and their relationship and influence in their communal and territorial environment. Part of the process finds support in the practical application of their ancestral experiences and knowledge, as a support for the creation of new knowledge at the family level, and its transfer and exchange from the agroecological families to the local producers themselves, using educational methodologies andragogic. The objective of the study is to analyze the level of relationship and influence between the educational experience in family agroecological production with the agricultural development processes of the families of the Llañucancha community, district and province of Abancay, Apurimac region. For the study, the methodological design was applied: "NonExperimental Correlational".
\end{abstract}

Keywords: Family farm unit, agriculture, livestock. 


\section{INTRODUCCIÓN}

Para complementar el análisis de los enfoques relacionados a la temática del estudio de investigación, se tomó en cuenta estudios previos realizados en diferentes ámbitos, de los cuales se tiene resultados concretos que contribuyen al planteamiento y definición de nuestras hipótesis; estos alcances se mencionan de manera respectiva o correspondiente en la cita de autores de la sección de Resultados, tales como: Carmen Rivera. 2012, concluye que, el conocimiento campesino debe generar encuentros entre ellos, los cuales, en base a una adecuada comprensión, tengan que ser plasmadas en procesos de practica agrícola sustentable a partir del desarrollo e intercambio entre campesinos.

Asimismo, Moura, Elías. 2005, pone énfasis en procesos de agricultura familiar desde enfoques agroecológicos de producción, donde las estrategias de capacitación, enfoque de género, y validación de las experiencias, forman parte del aporte y resultados de su estudio.
A su vez Isidro, Ramón. 2004, con su estudio sobre caracterización del potencial agroecológico de familias productoras; nos remite a reconocer que los modelos diversificados de producción familiar mantienen su arraigo, y que éstos sistemas productivos, constituyen un factor determinante para los procesos de desarrollo territorial.

Por tanto, estos estudios -en base a sus resultados-, generan vinculación con nuestro tema de investigación, y cuyo relacionamiento y enfoques guardan contribuyeron a la definición de criterios y análisis de resultados de nuestro estudio.

Los alcances de las líneas precedentes, derivan de algunos elementos $y$ documentación que formaron parte de la revisión y que guardan relación con el tema de estudio, fueron consideradas por su relación con el tema de investigación. A saber:

Rivera, Carmen. 2012; en su tesis, para optar el grado académico de Doctor en la Universidad del Valle (Colombia), titulada "Encuentro de maneras de conocer en el enfoque agroecológico”, 
tuvo por objetivo comprender esas maneras de conocer del campesino, asimismo establecer qué caracteriza el conocimiento de los que están dispuestos a encontrarse para desarrollar conjuntamente prácticas agrícolas sustentables, describir cómo son las relaciones que se desarrollan a partir de este encuentro e identificar las marcas que esta interlocución deja en el conocimiento del otro.

Moura, Elías. 2005; en su tesis, para optar el grado académico de Doctor en la Universidad de Córdoba (España), titulada “Análisis, desde la Perspectiva Agroecológica, de los Cambios Generados por un Proyecto de Desarrollo Rural en Agricultura Familiar: El Caso del Proyecto Gavião, Bahía - Brasil”, cuyo objetivo es analizar, desde la perspectiva agroecológica, los cambios generados por el Proyecto Gavião, midiendo, comparando y evaluando los resultados de las acciones ejecutadas en el área de su actuación, asimismo conocer los resultados de las acciones de asistencia técnica, validación de tecnologías, capacitación, organización, financiamiento de la producción agrícola, enfoque de género, protección del ambiente e implantación de obras de infraestructura.
Isidro, Ramón. 2004; en su trabajo de investigación, para la Universidad Nacional de La Plata (Argentina), titulado “Caracterización del potencial agroecológico en productores familiares: Un estudio en la Cuenca del Salado, Argentina”, tuvo por objetivo conocer el potencial agroecológico de los sistemas productivos familiares como aporte a una estrategia de desarrollo sustentable regional, asimismo conocer el manejo productivo en las unidades de producción familiares. En los resultados se destacan que la esencia de los modelos diversificados con base en el trabajo familiar, aún se mantienen en esta región. En este sentido, la "marginación" que ha sufrido esta región ha favorecido una relativa preservación del patrimonio cultural y natural.

\section{DESARROLLO METODOLÓGICO}

El Método aplicado en el estudio de investigación, fue el diseño Descriptivo Correlacional No Experimental, pues no se busca la relación de causalidad que existe entre las variables en estudio.

El diseño para el estudio de investigación es de corte transversal y de tipo comparativo, ya que tiene como 
propósito verificar si existen o no diferencias significativas con respecto a la variable que se mide en dos grupos diferentes.

El método con mayor influencia para el estudio fue el "hipotético deductivo" que, según Popper (Citado por Mejía, E. 2008. p 16); procedimiento que contribuyo al proceso lógico del pensar conducente a complementar la tarea investigativa del estudio, con fines de producir mayores y nuevos conocimientos en la temática del estudio.

Los instrumentos para la recolección de datos fueron los "Cuestionarios", de tipo abierto y con escala de valoración; los mismos que se estructuraron a través de fichas técnicas diseñadas para cada variable y contaron la respectiva "Validación de Expertos" conformado por docentes de la UNMSM.

Las variables de estudio fueron: Variable X: Experiencia de Educación en Producción Agroecológica Familiar; y Variable Y: Desarrollo Agropecuario.

Considerando que, cuando se analizan coeficientes, se asocian valores o niveles numéricos o escalares, éstos permiten cuantificar el grado de ajuste y de relación lineal entre dos variables; para nuestro caso el proceso de sistematización de la información recopilada, conto con el procesamiento de datos a través del paquete computacional SPSS, y su análisis correlacional, con el "Coeficiente de Correlación de Sperman" (Díaz Ignacio; et al. 2014. p 16); para el relacionamiento de las variables de estudio, en base a la sistematización de los 26 ítems del cuestionario, comprendidos en las 6 dimensiones del estudio.

El universo poblacional estuvo constituido por las 70 familias que actualmente están asentadas y empadronadas en la comunidad campesina de Llañucancha, distrito y provincia de Abancay; considerada ésta como una población homogénea. El tamaño de la muestra, se definió por el "principio de saturación de datos", criterio que considera un nivel de informantes en donde ya no se obtiene información nueva; y precisamente este nivel se alcanzó con 30 familias. Por tanto, el tamaño de la muestra para nuestro estudio fue de 30 unidades agrícolas.

Las "unidades de análisis" que estuvo conformado por los "productores(as)" correspondientes a las "unidades típicas", que, por ser homogéneas y de 
carácter finito; conllevo a definir a su vez a las "unidades de muestreo" (70 familias empadronadas), que vendrían a ser las "unidades agrícolas"; y que representan a las familias que desarrollan actividades productivas en la comunidad.

Por el tipo de estudio, se aplicaron procedimientos de muestreo "No Probabilístico", o también llamadas dirigidas; $y$ en base a algunas características definidas de interés para el estudio y definidas por el investigador, que suponen un procedimiento de selección informal y un arbitrario; son utilizadas en muchas investigaciones, sobre todo las que requieren la selección de sujetos con una determinada característica, especificadas en el planteamiento del problema. (Monje A. Carlos A. 2011. p 128).

El tamaño de la muestra, por las características propias del ámbito de estudio, se determinó en base a las necesidades de información, aplicando el "principio de saturación de datos", el mismo que permitió alcanzar un nivel donde ya no se obtiene nueva información, o ésta comienza a ser redundante (Monje A. Carlos A. 2011. p 131). Para nuestro estudio, este nivel se alcanzó con una unidad de análisis de 30 productores(as) agroecológicos.

Sin embargo, considerando a la población total comunal como "finita" y homogénea, es posible calcular el tamaño de la muestra utilizando la fórmula de muestra poblacional, considerando para ello a: $\mathbf{n}=$ tamaño de la muestra; $\mathbf{N}=$ población total; $\mathbf{Z}=$ nivel de confianza; $\mathbf{d}=$ margen de error; $\mathbf{p}=$ probabilidad a favor; $\mathbf{q}=$ probabilidad en contra. (Fuentes-Doria, Deivi D.; et al. 2020. p 63).

\section{RESULTADOS}

La prueba de Hipótesis General, se halló mediante la correlación de Spearman (correlación muy buena; Rho $=0.861$ ), y una significancia; $p=0,000$, por lo cual, se rechazó la hipótesis nula y se aceptó la hipótesis alterna. Se encontró que en la Variable $\mathbf{X}$ : Experiencia de Educación en Producción Agroecológica Familiar, el $86.7 \%$ manifiestan estar satisfechos; y en cuanto a la Variable Y: Desarrollo Agropecuario, el $90.0 \%$ también manifiestan estar satisfechos.

La validación externa de los instrumentos para las Variables $\mathbf{X}$ e $\mathbf{Y}$ (valoración de $\mathbf{9 1 . 8 3 \%}$ y $\mathbf{9 2 . 4 2 \%}$ respectivamente), se califican como muy adecuadas, de alta calidad y 
confiabilidad para el recojo de información primaria.

Estos resultados corroboran que entre las variables estudiadas (Variable $\mathbf{X}$ : Experiencia de Educación en Producción relación pertinente entre los procesos de intercambio y su influencia, que promueven los productores agroecológicos sobre los productores que desarrollan sistemas convencionales Agroecológica Familiar; y Variable Y:

Desarrollo Agropecuario), existe una

\begin{tabular}{|c|l|r|r|r|r|}
\hline \multicolumn{2}{|c|}{$\begin{array}{c}\text { Experiencia de Educación en } \\
\text { Producción Agroecológica Familiar }\end{array}$} & Frecuencia & Porcentaje & \% Válido & \% Acumulado \\
\hline \multirow{4}{*}{ Válidos } & Deficiente & 1 & 3.3 & 3.3 & 3.3 \\
\cline { 2 - 7 } & Satisfactorio & 26 & 86.7 & 86.7 & 90 \\
\cline { 2 - 7 } & Muy Bueno & 3 & 10 & 10 & 100 \\
\cline { 2 - 7 } & Total & 30 & 100 & 100 & \\
\hline
\end{tabular}

Cuadro 2: Contraste Hipótesis-Paquete Computacional SPSS 21.

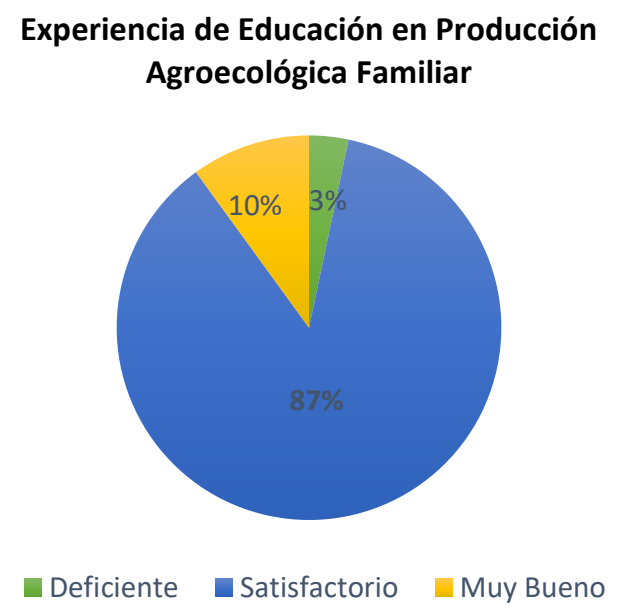

Grafico 1: Contraste de Hipótesis.

\section{CONCLUSIONES - DISCUSIÓN}

Los procesos de transferencia e intercambio de experiencias entre las familias rurales, a través de las escuelas de campo (ECAs), la metodología de enseñanza de campesino a campesino $(\mathbf{C a C})$, en base a los saberes locales existentes y validados por algunas familias en torno a la producción agroecológica, constituyen una de las herramientas de generación de cambio 
dentro de los procesos de desarrollo rural.

Un contraste con los antecedentes de investigación señalados anteriormente, muestran algunas concordancias con los resultados de nuestra investigación; así, de manera resumida mencionamos a Rivera, Carmen. 2012; que considera que para un desarrollo agropecuario en la comunidad es importante contar con los conocimientos campesinos y de los investigadores agroecológicos, de esta manera obtener mejores resultados en beneficio de la realidad agrícola y pecuaria.

Asimismo, los alcances del análisis de Moura, Elías. 2005, permite inferir sobre la importancia de los procesos de réplica y resultados, luego de implementar sistemáticamente algunos cambios en la agricultura familiar y la validación de las mismas, desde una estrategia de producción agroecológica.

De igual manera los resultados obtenidos por Isidro, Ramón. 2004, incide en que los potenciales agroecológicos de los sistemas productivos familiares representan un gran aporte a la estrategia de desarrollo sustentable regional.

Con el estudio se determinó que, bajo buenos procesos de Experiencia de
Educación en Producción Agroecológica Familiar, se genera cambios en los procesos de desarrollo agropecuario, con influencia favorable sobre los cambios de actitud y valoración de los saberes locales para el desarrollo de una "agricultura limpia".

A nivel de efecto: Las familias del ámbito comunal de estudio, -en un proceso sistemático-, vienen comprendiendo que la educación e intercambio de experiencias validadas en producción agroecológica, conlleva a mejores escenarios productivos.

A nivel de impacto: Se asume que, en el mediano plazo, bajo sostenidos procesos y estrategias de gestión y producción agroecológica, se estará incidiendo en el mejoramiento de la calidad de vida de las familias rurales, logrando influenciar en los procesos productivos a nivel local y regional; y su contribución a los procesos comunales e institucionales para la seguridad y soberanía alimentaria.

Se destaca la importancia del análisis del modelo agroecológico de los sistemas productivos familiares, comprendiendo que esos procesos resultan beneficiosos, pues adaptan, intercambian y articulan las labores de producción y conservación del ambiente con la cultura y saberes 
locales, hacia procesos de interacción entre las familias de la comunidad.

Los resultados de la investigación demuestran que existen tendencias hacia la predisposición de las familias de productores(as), para la aplicación sistemática de tecnología apropiada y validada a través de la creación y conservación de saberes locales, conducentes a procesos de innovación para el desarrollo sostenible de su agricultura y producción pecuaria.

REFERENCIAS (CITADAS EN EL ARTÍCULO)

- DÍAZ, Ignacio. et al. (2014). "Guía de Asociación entre variables (Pearson y Spearman en SPSS)". Ayudantía Estadística I. 19 pág. Universidad de Chile. FACSO.

- FUENTES-DORIA, Deivi D. et al. 2020. "Metodología de la Investigación” Conceptos, herramientas y ejercicios prácticos en las ciencias administrativas y contables. Editorial Universidad Pontificia Bolivariana. Primera Edición.

- MEJIA MEJIA, Elías (2008). “ $L a$ Investigación Científica en Educación". Lima. Centro de Producción Editorial e Imprenta de la UNMSM. Primera Edición.

- MONJE Álvarez, Carlos A. (2011). "Metodología de la Investigación Cuantitativa y Cualitativa". Neiva-Colombia. Programa Comunicación Social y Periodismo.
REFERENCIAS (CITADAS EN EL ESTUDIO DE INVESTIGACIÓN)

- ARIAS GAVIRIA, Jairo (2014). "Educación Rural y Saberes Campesinos en Tierradentro Cauca”. Estudio del Proceso Organizativo de la Asociación Campesina de Inzá Tierradentro (ACIT). Tesis Universidad Nacional de Colombia.

- BURGOS S., Diego A. (2010). "Agroecología y Saber Local" Bases de la Educación Popular para el Vivir Bien. Agroecología Universidad de Cochabamba. AGRUCO-Bolivia.

- MUJICA RIVERO, Henry; et al. (2015). "Aula Agroecológica. Alternativa de Enseñanza para Escuelas Rurales". Consejo de Investigación. Saber, Universidad de Oriente. Cumaná-Venezuela. Vol. $27 \mathrm{~N}^{\circ}$ 1: 120-129.

- NAVAS PANADERO, A.; VELÁSQUEZ MOSQUERA, Juan C. (2014). "Enfoque Sistémico en el Análisis de Sistemas de Producción Agropecuaria”. Una mirada más allá de lo disciplinar. Revista Ciencia Animal. N ${ }^{\circ}$ 7: 99110. Universidad de La Salle. Bogotá-Colombia.

- PIAMONTE, R. (1995). "Experiencias Latinoamericanas. Indicadores de sustentabilidad en agroecosistemas". En Alatorre Frenk y García Campos (comp.) Módulo I. EpistemológicoIntercultural. Sustentabilidad: Desarrollo regional sustentable.

- SALCEDO SALOMÓN y GUZMÁN Lya. (2014). "Agricultura Familiar en América 
Latina $y$ el Caribe: Recomendaciones de Política". Publicación de la Organización de las Naciones Unidas para la Agricultura y Alimentación.FAO.

- TOLEDO, V. M. (1995). "Agroecología, sustentabilidad y reforma agraria" La superioridad de la pequeña producción familiar. Vol. $3 \mathrm{~N}^{\circ}$ 2: 10. México.

- COMUNIDAD ANDINA (2011). "Agricultura Familiar Agroecológica Campesina en la Comunidad Andina”. http://www.comunidadandina.org/ Upload/2011610181827revista_agr oecologia.pdf.

- GONZÁLEZ, M. (2009). "Principales Tendencias y Modelos de la Educación Ambiental en el Sistema Escolar". Organización de Estados Iberoamericanos. http://www.oei.es/oeivirt/rie11a01. $\underline{\mathrm{htm}}$.

\section{GALERÍA}

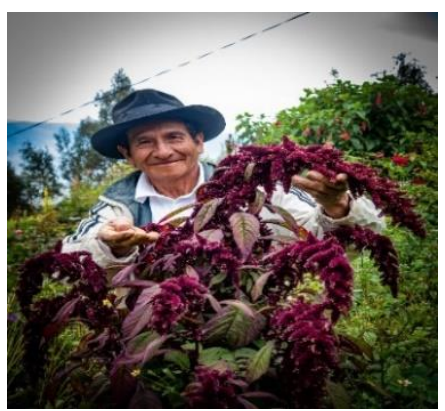

Don Santos Pineda Batallanos Productor agroecológico referente de nuestro estudio y de la Comunidad Llañucancha Abancay (Fundo Bello Paraíso)
- ISIDRO, Ramón (2004).

"Caracterización del Potencial Agroecológico en Productores Familiares". Un estudio en la Cuenca del Salado, Argentina. http://sedici.unlp.edu.ar/bitstream/h andle/10915/38758/Documento_co mpleto.pdf? sequence $=1$.

- MOURA, Elías (2005). "Análisis, desde la Perspectiva Agroecológica, de los cambios generados por un Proyecto de Desarrollo Rural en Agricultura Familiar". El Caso dei ProyectoGavião, Bahía - Brasil”. http://orgprints.org/24941/1/Reis_A n\%C3\%A1lisis.pdf.

- RIVERA, Carmen (2012). "Encuentro de Maneras de Conocer en el Enfoque agroecológico". Revisado el 23 de Julio del 2015. http://bibliotecadigital.univalle.edu. co/bitstream/10893/4714/1/CB0475891.pdf.

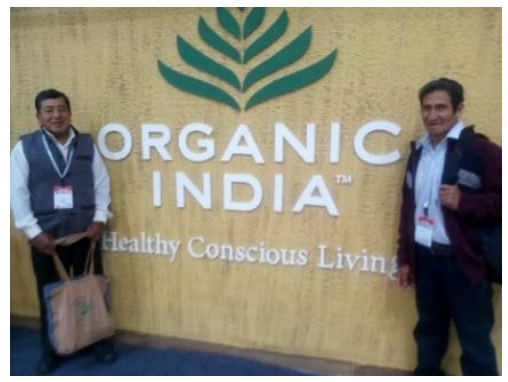

Representantes nacionales en evento agroecológico en el país de la India.

Derecha: Santos Pineda Batallanos (representante comunidad Llañucancha-Abancay) 